\title{
Current and novel pharmacological therapeutic ap- proaches in Post-Traumatic Stress Disorder. A brief review
}

\author{
Carmen-Maria Rusz ${ }^{1}$, George Jîtcă²,3*, Amalia Miklos, Mădălina-Georgiana Bătrînu³, Bianca Eugenia \\ Ösz², Silvia Imre ${ }^{5}$ \\ 1. Faculty of Pharmacy, George Emil Palade University of Medicine, Pharmacy, Science, and Technology of Targu Mures, Romania \\ 2. Department of Pharmacology and Clinical Pharmacy, Faculty of Pharmacy, George Emil Palade University of Medicine, Pharmacy, Science, and Technology \\ of Targu Mures, Romania \\ 3. Doctoral School of Medicine and Pharmacy, I.O.S.U.D., George Emil Palade University of Medicine, Pharmacy, Science, and Technology of Targu Mures, \\ Romania \\ 4. Department of Biochemistry, Faculty of Pharmacy, George Emil Palade University of Medicine, Pharmacy, Science, and Technology of Targu Mures, \\ Romania \\ 5. Department of Analytical Chemistry and Drug Analysis, Faculty of Pharmacy, George Emil Palade University of Medicine, Pharmacy, Science, and \\ Technology of Targu Mures, Romania
}

\begin{abstract}
Objective: Although not highly prevalent among the general population, post-traumatic stress disorder is a serious psychiatric condition, associated with co-morbidities, mortality and high suicide rates. Currently, there are few approved pharmacological therapies, which count as second-line, augmented to psychotherapy. Studies from the literature emphasize the need for novel treatment options, due to high relapse rates and patients that do not achieve remission. This study provides an overview over the pharmacological treatment of post-traumatic stress disorder, from a neurobiological perspective. Methods: A systematic research has been conducted through PubMed, PLOS one, Cochrane library and Google Scholar databases. Results: The neurobiological mechanisms which underlies the symptomatology are not fully elucidated. In the present, some theories involved in the onset/ manifestation are formulated (serotonergic, noradrenergic, glutamatergic, GABA-ergic, endocannabinoid) and the current therapy aims to modulate these neurotransmissions. In light of the studies along the years, a line should be drawn between the drugs acting on reducing the anxiety only and those that exhibit dual effect i.e. reducing the anxiety and affecting the memory reconsolidation processes. Although labelled as recreational drugs rather than compounds with intended therapeutic effects, cannabidiol and 3,4-methylenedioximethamphetamine appear to be the most promising from the perspective of efficacy and benefitrisk ratio. Conclusion: Preclinical studies come with acceptable results, yet clinical trials are controversial and heterogeneous, given the small population size. Given the seriousness of post-traumatic stress disorder, the attempts to find effective and safe treatment in a context that lacks appropriate therapeutic approaches should be encouraged.
\end{abstract}

Keywords: PTSD, treatment, antidepressants, cannabidiol, 3,4-methylenedioximethamphetamine

Received 26 June 2021 / Accepted 26 August 2021

\section{Introduction}

Post-traumatic stress disorder (PTSD) is one of the very complex psychiatric conditions that can be triggered upon exposure to traumatic events, such as wars, human cruelty, natural disasters, life-threatening accidents and its symptoms include irritability, nightmares, poor concentration, hypervigilance and emotional withdrawal [1]. This condition causes physical and mental impairments and it is associated with co-morbidities such as depression and other anxiety disorders, high mortality and high suicide rates. PTSD could affect any individual at a particular moment in life-time, from early childhood to late adulthood, due to the high prevalence of traumatic event occurrence during lifetime.

In the global context, the prevalence of PTSD is highest among the veterans, the population living in low or middle-income countries, people in zones of conflict [1, 2]. According to World Mental Health Surveys on Trauma

${ }^{*}$ Correspondence to: George Jitcă

E-mail: george.jitca@umfst.ro and PTSD, $70 \%$ of the respondents stated that they were exposed to a traumatic event. Of these, the most frequent reported were violence/ conflicts, witnessed accidents, lifethreatening injuries or illnesses, torture. Interestingly, these surveys revealed that most of the traumas occurred early in life $[3,4]$. With regards to the clinical picture, the complex symptomatology is attributed to the emotional dysregulation which divide PTSD into two contrasting forms that fluctuate between one another: emotional under-modulation (hyperarousal) associated with re-experiencing the traumatic episode, fear, anger, guilt and emotional overmodulation (hypoarousal), associated with depersonalization, avoidance of traumatic triggers, sleep disturbances, emotional numbing and analgesia [1].

First-line therapy consists in psychotherapy which focuses on targeting and diminishing the fear-based emotional reactions. There have been identified two types of approaches: trauma-focused and non-trauma focused therapy. Trauma focused therapies: Cognitive behavioral therapy, Prolonged-exposure therapy and Cognitive Processing therapy are centered around the idea that promoting 
emotion regulation and extinction learning will result in diminishing the distress caused by trauma-related thoughts and the avoidance behavior. Almost comparable from efficacy perspective, but holding the advantage of lower dropout rates, non-trauma focused therapies (present-centered therapy, interpersonal therapy) have wider focus on meaning making or addressing current life stressors or goals [1]. Psychotherapy usually consists in weekly sessions, with a duration of 6 to 16 weeks as a global standard [1]. With regards to the pharmacotherapy, which is augmented to the psychotherapy approach, an increasing need for novel treatment options is emphasized by many authors in the literature, mainly due to PTSD characteristics of genotypic and phenotypic heterogeneity, failed studies, chronicity, relapse, and the high number of patients who do not achieve remission [5-8].

Currently, only sertraline and paroxetine received Food and Drug Administration's (FDA) approval for the indication of PTSD. Among these, evidence-based guidelines recommend the use of fluoxetine and venlafaxine as monotherapy for the patients that are unable to access traumafocused psychotherapy $[9,10]$. Nonetheless, novel pharmacotherapy agents are promising in relieving one or more symptoms, by addressing the complex biological targets and molecular pathways involved in the pathological response.

The purpose of the present study is to provide an insight over the PTSD novel pharmacological psychoactive agents and their potential benefits in relation with their neurobiological implications. Efforts have been made over the past years in order to understand the mechanisms underlying the onset and symptomatology of PTSD. The main brain areas involved in the fear and stress response are: the amygdala, the prefrontal cortex (PFC), nucleus accumbens (Nac), the hypothalamus and hippocampus (glucocorticoid targets). A "neurocircuitry model" of PTSD has been proposed by Rauch et al., [11, 12]. According to the authors, in PTSD, acute changes in brain activity are incriminated, such as a deficit in the extinction of fear-conditioning mediated by the amygdala, associated with increased activity in this area, decreased activation of the ventral/ medial PFC along with a reduced hippocampal activity and volume $[11,12]$. Among these brain activity modifications, neurobiological imbalances are also involved: increased inflammatory response mediated by the hypothalamic pituitary adrenal (HPA) axis, altered neurobiological pathways covered by monoaminergic, serotonergic, dopaminergic, glutamatergic, GABA-ergic, endocannabinoid systems and cognitive deficits associated with these pathways in several brain areas [13]. Although the interconnections established are not fully understood yet, some mechanisms are proposed in the attempt of elucidating the PTSD dilemma. The strong connection between the monoaminergic dysregulation and PTSD has been emphasized by many studies. Increases in catecholamine lead to weak PFC synaptic connectivity and increased neuronal activity in the amygdala and Nac [14].
For the noradrenergic pathway, a high norepinephrine (NE) release has been associated with increased activity in the amygdala. High levels of NE disrupt the inhibition that normally, the PFC holds on the amygdala through the $\alpha_{1}$ receptors, therefore, the amygdala-defensive behavior characteristic in PTSD is activated [15-17]. Consequently to the activation of amygdala, a hippocampal increased activity has also been observed, leading to the hypothesis that these two brain structures co-activate at the exposure of the individuals to negative images or when accessing their personal traumatic memories, in comparison with nontraumatized controls. Moreover, a less engagement of these two structures has been indicated as a response to positive images for PTSD patients (for a review, see Fitzgerald et al., [18]). A schematic representation of the main brain regions affected by PTSD is shown in Figure 1.

\section{Material and methods}

Systematic research has been conducted through PubMed, PLOS one, Cochrane library and Google scholar databases. The search terms used were: 'PTSD', 'onset', 'treatment', 'neurobiology'.

\section{Discussion}

Extensive possible explanations in how the aforementioned systems could interfere with the onset of PTSD were offered by the literature. However, controversial results have been obtained, as the involvement of a certain system is not always supported by treatment studies. For exemplification purposes, the discussion will be carried out for some of these mechanisms and their approach in PTSD.

Several studies have demonstrated the linkage between the dysregulation of the HPA axis and PTSD onset, mainly throughout the low levels of cortisol secretion, which further points to vulnerability to develop PTSD $[19,20]$. In addition to glucocorticoid's ability to modulate the stress

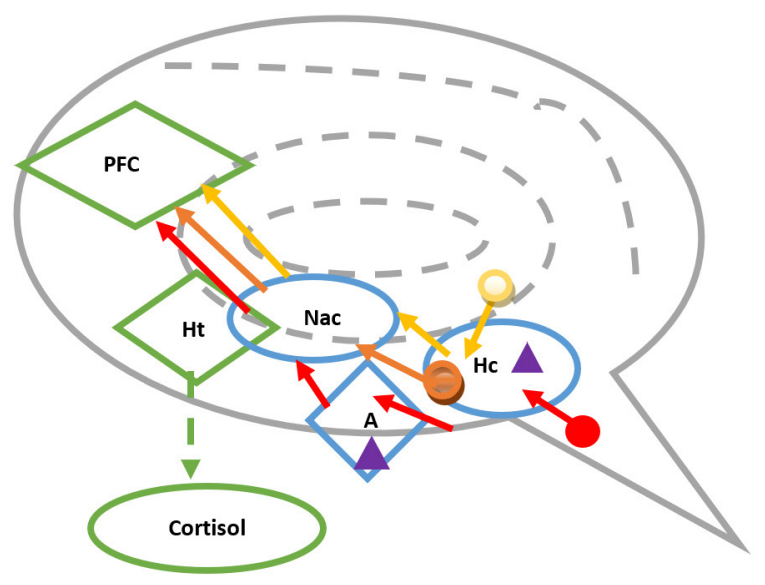

PFC - prefrontal cortex; $\mathrm{Ht}$ - hypothalamus; $\mathrm{Nac}$ - nucleus accumbens; $\mathrm{Hc}$ - hippocampus; A - amygdala; The orange, yellow and red arrows represent the monoaminergic pathways/ projections (red - norepinephrine, orange - serotonine and yellow - dopamine). The purple triangles are attributed to the cannabinoid receptors CB1, which are highly expressed in the amygdala and hippocampus.

Fig. 1. Schematic representation of the main brain regions affected by PTSD 
response, its involvement in memory encoding/ retrieving is suggested [21]. Low-levels of cortisol have been detected in different samples early after trauma exposure [22]. Studies indicate that immediate administration of cortisol after the traumatic episode exposure might reduce the possibility of developing PTSD $[23,24]$. A systematic review conducted by Speer et al., [21] concludes that regardless the dysregulation of HPA axis in PTSD, further consideration must be done in the exact manner in which cortisol is involved. This could be achieved by standardization of study designs [21].

Serotonergic system, together with noradrenergic and dopaminergic systems play a key role in modulation of different psychological and behavioral functions. These neurotransmissions overlap in some specific brain areas, as they share the same transporter and they modulate one another. While dopaminergic transmission relates to the reward function, NE and serotonin (5-HT) modulate stress response, anxiety, mood and emotional response.

The implications of serotonergic neurotransmissions in PTSD are related to the 5-HT projections in key areas of fear circuitry in amygdala, hippocampus, $\mathrm{PFC}$ and also the GABA-ergic inhibitory neurons. In preclinical studies, it has been demonstrated the increase of 5-HT secretion and release as a response to a stress factor. Another important input comes from the serotonergic receptors; the administration of a $5-\mathrm{HT}_{1 \mathrm{C}}$ agonist, meta-phenylchloropiperazine led to panic attacks and anxiety onset in PTSD patients [25], for a review see Kelmedi et al., [26], while preclinic studies demonstrate the fact that in the absence of $5-\mathrm{HT}_{1 \mathrm{~A}}$ receptors in mice, the anxiety levels and fear response are exacerbated [27]. These findings resulted in the theory that $5-\mathrm{HT}_{1 \mathrm{~A}}$ agonists might be considered a therapeutic option. Other preclinical studies find that $5-\mathrm{HT}_{1 \mathrm{~B}}$ field receptor density is reduced in key areas (amygdala, PFC and cingulate gyrus) upon stress exposure. These results are supported by Positron Emission Tomography (PET) scans in PTSD patients [28]. Based on their anxiolytic effects, selective serotonin reuptake inhibitors (SSRIs) are recommended as second-line treatment, being effective in decreasing hyperarousal symptoms of PTSD (irritability, anger, depression) but having little effect on hypoarousal symptoms $[6,19]$. When administered shortly after exposure to trauma, SSRIs - escitalopram, showed promising response in acute reduction of PTSD symptoms [29].

According to Krystal and Neumeister [5], a strong correlation exists between NE and PTSD, given the hyperadrenergic symptoms that some patients may experience (hyperarousal form). Moreover, the same author suggests that the NE transporter (NET) is dysregulated in PTSD, as in preclinical studies, chronic stress modulates the NET availability in the synaptic cleft, which may lead to an exaggerated synaptic availability of $\mathrm{NE}$ in projection areas and dopamine (DA) in the frontal cortex. Thus, NET could constitute an important marker for diagnosis of PTSD $[5,30]$, especially taking into the consideration that NET blockers are successfully used since many years for treating anxiety and depressive disorders. Another suggested implication is related to the density of $\alpha_{2}$ presynaptic receptors, which appears to be lower in PTSD patients. Yohimbine, an $\alpha_{2}$ antagonist lowered the metabolic activity in the PFC, generating panic attacks and recurrent memories $[31,32]$. Prazosin, an $\alpha_{1}$ adrenoreceptor antagonist, which initially showed promising results in treating sleep-related symptoms in PTSD, failed to demonstrate this effect in large randomized control trials $[33,34]$.

While another promising candidate - propranolol showed inhibition of consolidation of traumatic memories in preclinical models, clinical trials conducted do not support these findings [35]. Oxytocin, a neuro-hormone responsible for affective behavior, social cognition and interaction also represents a novel pharmacological target in the past years, as in stress-related disorders, it could improve symptoms such as anhedonia, psychosocial stress reactivity, fear and anxiety. In PTSD, oxytocin was most intensively studied in human subjects. Acute intranasal oxytocin administration is correlated with reduction of PTSD symptoms, supported by many studies from the literature [36, 37]. Moreover, oxytocin has been studied in augmentation to therapy based on its many beneficial effects, but mostly on the promoting therapeutic alliance, with promising results [38].

While the biological targets presented so far demonstrated some positive outcomes in the treatment of PTSD, the relapse, recurrence rate and discontinuation are high among the PTSD patients treated with these substances. Studies show that one of the reasons could be the low adherence to the treatment due to the side-effects. The glutamatergic pathway, which has strong implications in neuronal plasticity, learning process and memory formation via NMDA receptors, is also a key factor in PTSD onset. In animal models, it has been demonstrated that acute exposure to stress elevated the glutamatergic neurotransmission hence the glutamate levels in PFC, amygdala and hippocampus, and also a modification of receptor density in hippocampus [39]. Clinical studies support this theory, as high levels of glutamate have been identified in PTSD patients examined through Magnetic Resonance Spectroscopy (MRS) [40]. Gamma-aminobutyric acid (GABA) is a neurotransmitter involved in memory and emotional encoding and fear response. With regards to GABA neurotransmission, it has been suggested that in PTSD, low levels of GABA are incriminated, which are strongly related to low stimulation of benzodiazepine receptors [26].

In light of the studies from the literature along the years, a line should be drawn between the drugs acting on reducing the anxiety only and the ones that exhibit dual effect i.e. reducing the anxiety and affecting the memory reconsolidation processes. Although labelled as recreational drugs rather than compounds with intended therapeutic effects, cannabidiol (CBD) and 3,4-methylenedioximethamphetamine (MDMA) appear to be the most promising 
in this area, from the perspective of the efficacy and benefit-risk ratio.

The endocannabinoid system, which modulates fear, anxiety and stress-related neuronal activity has been intensively studied for its major and complex involvement into the emotional and cognitive responses. Although cannabis effects on mood i.e. relaxation and elevated mood is well established by its recreational use mostly due to the $\Delta$ 9-trans-tetrahydrocannabinol $(\triangle 9$-THC) component, the exact mechanism responsible for PTSD symptomatology remains to be fully elucidated. It has been suggested that from the two most important components of cannabis, $\triangle 9$-THC and CBD, the latter would be potentially responsible for the benefic effects on PTSD, in part for interfering with the serotonergic system [41]. Moreover, unlike $\triangle 9$-THC, CBD does not present the potential for drug abuse, as shown by preclinical studies.

As such, cannabidiol has been researched for its potential in several psychiatric disorders, with focus on depression and PTSD; while studies on animals subjected to stress models assessed the anxiety like-behavior and its involvement in fear-memory with positive outcomes [42, 43], human studies suggest reduction of PTSD severity $[44,45]$ and positive results in both fear extinction and memory encoding processes $[45,46]$, (for a review see Bitencourt et al., [47]). According to the World Health's Organization's review report on CBD, it has a good safety profile with limited side effects $[48,49]$. The anxiolytic activity of CBD is mainly indirect, as CBD is, according to several studies, a weak agonist of both $\mathrm{CB} 1$ and $\mathrm{CB} 2$ receptors [44]. The interaction with these receptors is complex, as CB1 receptors are also located in the glutamatergic and GABA-ergic neurons. The endocannabinoid ligands, anandamide and 2-arachidonoylglycerol (2-AG) indirectly controls DA release via glutamatergic and GABA-ergic neurons: they enhance GABA release and inhibit the glutamatergic release, which finally translates into the normalization of dopaminergic neurotransmission. A deficit in endocannabinoids would lead to the loss of inhibitory control of GABA on DA release, resulting in a dopaminergic disinhibition - for a review see Kelmendi et al., [26].

However, due to its many biological targets, it has been suggested that CBD effect is attributed partly to the interaction with the serotonergic pathway (activation of the $5-\mathrm{HT}_{1 \mathrm{~A}}$ subtype of serotonergic receptors) as well as with the corticosteroid one (the endocannabinoid pathway exerts an inhibitory control on the HPA axis). CBD is responsible for the inhibition anandamide (one of the main ligands) reuptake and concomitantly, for the inhibition of fatty acid amide hydrolase (FAAH), an enzyme responsible for anandamide degradation. The inhibition of FAAH by CBD and consequently the increase of anandamide levels could explain its role in aversive memories [44, 47].

Nabilone, a synthetic analogue of $\triangle 9-\mathrm{THC}$ was also studied in this context, showing significant improvements in the quality of sleep and reduction of nightmares [50,
51]. Nonetheless, currently, there are no approved medication containing cannabis derivatives such as $\mathrm{CBD}$ and $\triangle 9$-THC. The only drug product containing CBD that received FDA approval is indicated in intractable childhood-onset seizures, the rest of the products that are found on the market are food supplements with limited quality control and unknown bioavailability. The question is also raised around the $\mathrm{CBD}$ liability of abuse, as some patients reported "pleasant drug effect" [49, 52]. Given its chronic dosage regimen in the treatment of PTSD, this aspect cannot be disregarded.

Addressing the complex mechanisms underlying PTSD onset, MDMA shows promising results in animal studies, in terms of fear extinction [53]. MDMA-assisted psychotherapy that has been intensively studied as a novel pharmacological substance by the Multidisciplinary Association for Psychedelic Studies (MAPS) obtained breakthrough therapy designation (BTD) by FDA in 2017. This has been granted by demonstrating the advantage in the primary endpoint, Clinician-Administered PTSD Scale (CAPSIV) score over Paroxetine and Sertraline and also a better safety profile reflected by reduced drop-out rates [54]. In this context, the authors of MAPS conducted the first randomized controlled pilot study (phase II trial), assessing the efficacy and safety of MDMA-assisted psychotherapy. Their findings indicate the fact that this pharmacological approach, augmented limited number of MDMA doses to patients undergoing psychotherapy, leads to statistically and clinically improvement of PTSD symptoms. The primary end-point of the treatment was measuring the total (CAPS-IV) score, which was significantly reduced compared to placebo [55]. Subsequently, the long-term followup of the subjects involved in the trial was performed by the same group of authors. The first, referring to the original study (range 17-74 months; mean of 45.4 months) reported sustained improvement of the symptoms without any associated cases of drug abuse afterward the treatment or neurocognitive decline [56]. The second one refers to a longitudinal-pooled analysis of six phase II trials taking place in different locations (12-month long-term followup) and reported clinically significant symptom improvement reflected in reduction of CAPS-IV score from treatment exit for $82 \%$ of the subjects [57] (for an extended review, see Illingworth et al., [58]).

MDMA, an amphetamine derivative, has complex pharmacological effects, mediated by a dual mechanism i.e. stimulation of 5-HT release and in the meantime inhibition of monoamine transporters, with particularly strongest affinity for serotonin transporter [58]. Additionally, it is known to increase the oxytocin levels in key regions of the brain. These effects translate into reduced anxiety, enhanced mood. MDMA is popular for its entactogenic effects and related to the increased oxytocin levels, there appears to be an effect of increased trust, which allows the patient to build a relationship based on trust with its psychotherapist. This particular connection is im- 
portant because it allows the patient to open up and face the repressed traumatic memories with a reduced sense of painful emotions, anxiety and without dissociation or emotional numbing $[55,59,60]$.

An important aspect brought into attention regarding the MDMA-assisted psychotherapy is that it should not be confounded with a promising treatment for PTSD, but as a process facilitator among psychotherapy. Moreover, patients targeted for this approach are the ones that are facing treatment-resistance and the unsuccessful psychotherapy, due to their inability to open up over time. Some important reasons are related to the fact that psychotherapy requires long-term commitment with regular therapy sessions, to the difficulty of some patients to access their traumatic memories or to deal with the emotions that are triggered with the retrieval of the traumatic memories. These factors can provoke such distress to the patients that they are unable to continue with the psychotherapy [60]. More frequent side effects have been reported in case of MDMA use than in case of CBD: dry mouth, nausea, anxiety, inability to urinate, bruxism, restlessness, sleep disturbances, depression [59] increase in heart rate, blood pressure and in body-temperature and at higher doses, potentially fatal serotonin syndrome. The latter side effects can be attributed to the increased serotonin levels, which can be indirectly correlated with oxidative stress via $5-\mathrm{HT}_{2 \mathrm{~A}}$ receptors activation which leads to accumulation of DA in Nac $[58,61]$.

Moreover, in acute administration at higher doses, the metabolic pathway which consists in saturable enzymes, is also incriminated in generating neurotoxic species [62]. Studies show that acute administered MDMA in equivalent doses to street dose ecstasy lead to increase in neurotoxicity. It should be noted that these doses are comparable to the ones used in the MDMA-assisted psychotherapy studies [59].

\section{Conclusions}

Although recent studies demonstrate that some of these drugs exhibit on the overall reduction of PTSD symptomatology, the main emphasized aspect is the heterogeneity of the study designs, reported data and small population size in clinical trials. Extensive thorough studies remain of an utmost importance, especially addressing the concern of side effects of MDMA and liability of drug abuse in case of MDMA and CBD. Nevertheless, given the seriousness of PTSD and its association with increased morbidity, mortality and suicide risk, the attempts to find effective and safe treatment in a context that lacks appropriate therapeutic approaches should be encouraged.

\section{Author's contribution}

CMR - Conceptualization, Writing the original draft, Editing

GJ - Conceptualization, Writing and Editing

AM - Writing and Editing

MGB - Writing and Editing
BEÖ - Editing, Conceptualization, Supervision

SI - Editing, Conceptualization, Supervision

\section{Conflicts of Interest}

The authors declare no financial or other conflict of interest.

\section{References}

1. Yehuda R, Hoge CW, McFarlane AC et al. Post-traumatic stress disorder. Nat Rev Dis Primers, 2015, 1:15057.

2. Yehuda R, Lehrner A, Rosenbaum TY. PTSD and Sexual Dysfunction in Men and Women. J Sex Med, 2015, 12(5):1107-19.

3. Watson P. PTSD as a Public Mental Health Priority, Curr Psychiatry Rep, 2019, 21(7):61.

4. Benjet C, Bromet E, Karam EG, Kessler RC, et al. The epidemiology of traumatic event exposure worldwide: results from the World Mental Health Survey Consortium. Psychol Med. 2016, 46(2):327-43.

5. Krystal JH, Neumeister A. Noradrenergic and serotonergic mechanisms in the neurobiology of posttraumatic stress disorder and resilience. Brain Res, 2009, 1293:13-23.

6. Stein DJ, Ipser JC, Seedat S. Pharmacotherapy for post traumatic stress disorder (PTSD). Cochrane Database Syst Rev, 2006, 2006(1):CD002795.

7. Davidson JR. Remission in post-traumatic stress disorder (PTSD): effects of sertraline as assessed by the Davidson Trauma Scale, Clinical Global Impressions and the Clinician-Administered PTSD scale. Int Clin Psychopharmacol, 2004, 19(2):85-7.

8. Dieperink M, Erbes $\mathrm{C}$, Leskela $\mathrm{J}$ et al. Comparison of treatment for posttraumatic stress disorder among three Department of Veterans Affairs medical centers, Mil Med, 2005, 170(4):305-8.

9. Bernardy NC, Hoge CW, \& Friedman MJ. VA/DoD Clinical Practice Guideline for the management of posttraumatic stress disorder and acute stress disorder. Washington, DC: United States Departments of Veterans Affairs and Defense, 2017, available at VA/DOD Clinical Practice Guideline for the Management of Posttraumatic Stress Disorder and Acute Stress Disorder, accessed on 9th of June, 2021.

10. American Psychological Association. (2017), Clinical practice guideline for the treatment of posttraumatic stress dis order (PTSD) in adults. American Psychological Association Guideline Development Panel for the Treatment of PTSD in Adults, available at: https://www.apa.org/ptsdguideline/ptsd.pdf, accessed on 9th of June 2021.

11. Rauch SL, Shin LM, Phelps EA. Neurocircuitry models of posttraumatic stress disorder and extinction: human neuroimaging research-past, present, and future. Biol Psychiatry, 2006, 60(4):376-82.

12. Rauch SL, Shin LM, Whalen PJ, Pitman RK. Neuroimaging and the neuroanatomy of PTSD. CNS Spectr, 1998, 3(suppl 2):30 - 41.

13. Horn SR, Charney DS, Feder A. Understanding resilience: New approaches for preventing and treating PTSD. Exp Neurol, 2016, 284(Pt B):119-132.

14. Abdallah CG, Averill LA, Akiki TJ et al. The Neurobiology and Pharmacotherapy of Posttraumatic Stress Disorder. Ann Rev Pharmacol Toxicol, 2019, 59:171-189.

15. Rasmusson AM, Pineles SL. Neurotransmitter, Peptide, and Steroid Hormone Abnormalities in PTSD: Biological Endophenotypes Relevant to Treatment. Curr Psychiatry Rep, 2018, 20(7):52.

16. Arnsten AF. Stress signalling pathways that impair prefrontal cortex structure and function. Nat Rev Neurosci, 2009, 10(6):410-22.

17. Birnbaum S, Gobeske KT, Auerbach J, Taylor JR, Arnsten AF. A role for norepinephrine in stress-induced cognitive deficits: alpha-1-adrenoceptor mediation in the prefrontal cortex. Biol Psychiatry, 1999, 46(9):1266-74.

18. Fitzgerald JM, DiGangi JA, Phan KL. Functional Neuroanatomy of Emotion and Its Regulation in PTSD. Harv Rev Psychiatry, 2018, 26(3):116-128.

19. Quinones MM, Gallegos AM, Lin FV, Heffner K. Dysregulation of inflammation, neurobiology, and cognitive function in PTSD: an integrative review. Cogn Affect Behav Neurosci, 2020, 20(3):455-480.

20. Pan X, Wang Z, Wu X, Wen SW, Liu A. Salivary cortisol in post-traumatic stress disorder: a systematic review and meta-analysis. BMC Psychiatry, 2018, 18(1):324. doi: 10.1186/s12888-018-1910-9.

21. Speer KE, Semple S, Naumovski N, D'Cunha NM, McKune AJ. HPA axis function and diurnal cortisol in post-traumatic stress disorder: A systematic review. Neurobiol Stress, 2019, 11:100180.

22. Yehuda R, Flory JD, Bierer LM et al. Lower methylation of glucocorticoid receptor gene promoter $1 \mathrm{~F}$ in peripheral blood of veterans with posttraumatic stress disorder. Biol Psychiatry, 2015, 77(4):356-64. 
23. Bandelow B, Baldwin D, Abelli $M$ et al. Biological markers for anxiety disorders, OCD and PTSD: A consensus statement. Part II: Neurochemistry, neurophysiology and neurocognition. World $\mathrm{J}$ Biol Psychiatry. 2017 Apr;18(3):162-214.

24. Zohar J, Yahalom H, Kozlovsky $\mathrm{N}$ et al. High dose hydrocortisone immediately after trauma may alter the trajectory of PTSD: interplay between clinical and animal studies, Eur Neuropsychopharmacol, 2011 (11):796-809.

25. Southwick SM, Krystal JH, Bremner JD et al. Noradrenergic and serotonergic function in posttraumatic stress disorder. Arch Gen Psychiatry, 1997, 54(8):749-58.

26. Kelmendi B, Adams TG, Yarnell S, Southwick S, Abdallah CG, Krystal JH. PTSD: from neurobiology to pharmacological treatments. Eur $J$ Psychotraumatol, 2016, 7:31858.

27. Ramboz S, Oosting R, Amara DA et al. Serotonin receptor 1 A knockout: an animal model of anxiety-related disorder. Proc Natl Acad Sci U S A, 1998, 95(24):14476-81.

28. Murrough JW, Czermak C, Henry $S$ et al. The effect of early trauma exposure on serotonin type $1 \mathrm{~B}$ receptor expression revealed by reduced selective radioligand binding. Arch Gen Psychiatry, 2011, 68(9):892-900.

29. Zohar J, Fostick L, Juven-Wetzler A et al. Secondary prevention of chronic PTSD by early and short-term administration of escitalopram: a prospective random ized, placebo-controlled, double-blind trial. The Journal of clinical psychiatry, 2018, 79(2):16m10730. doi: 10.4088/ JCP.16m10730.

30. Miner LH, Jedema HP, Moore FW, Blakely RD, Grace AA, Sesack SR. Chronic stress increases the plasmalemmal distribution of the norepinephrine transporter and the coexpression of tyrosine hydroxylase in norepinephrine axons in the prefrontal cortex. J Neurosci, 2006, 26(5):1571-8

31. Krystal JH, Webb E, Cooney NL et al. Serotonergic and noradrenergic dysregulation in alcoholism: m-chlorophenylpiperazine and yohimbine effects in recently detoxified alcoholics and healthy comparison subjects. Am J Psychiatry, 1996, 153(1):83-92.

32. Southwick SM, Krystal JH, Morgan CA et al. Abnormal noradrenergic function in posttraumatic stress disorder. Arch Gen Psychiatry, 1993, 50(4):266-74.

33. Raskind MA, Peskind ER, Chow Bet al. Trial of Prazosin for PostTraumatic Stress Disorder in Military Veterans. N Engl J Med, 2018, 378(6):507-517.

34. Germain A, Richardson R, Moul DE et al. Placebo-controlled comparison of prazosin and cognitive-behavioral treatments for sleep disturbances in US Military Veterans. J Psychosom Res, 2012, 72(2):89-96.

35. Brunet A, Saumier D, Liu A, Streiner DL, Tremblay J, Pitman RK. Reduction of PTSD Symptoms With Pre-Reactivation Propranolol Therapy: A Randomized Controlled Trial, Am J Psychiatry, 2018, 175(5):427-433.

36. van Zuiden M, Frijling JL, Nawijn L et al. Intranasal Oxytocin to Prevent Posttraumatic Stress Disorder Symptoms: A Randomized Controlled Trial in Emergency Department Patients. Biol Psychiatry, 2017,81(12):10301040.

37. Flanagan JC, Sippel LM, Wahlquist A, Moran-Santa Maria MM, Back SE. Augmenting Prolonged Exposure therapy for PTSD with intranasal oxytocin: A randomized, placebo-controlled pilot trial. J Psychiatr Res, 2018, 98:64-69.

38. Sippel LM, Allington CE, Pietrzak RH, Harpaz-Rotem I, Mayes LC, Olff M. Oxytocin and Stress-related Disorders: Neurobiological Mechanisms and Treatment Opportunities, Chronic Stress (Thousand Oaks), 2017, 1:247054701668799.

39. Pitman RK, Rasmusson AM, Koenen KC et al. Biological studies of posttraumatic stress disorder, Nat Rev Neurosci, 2012,13(11):769-87.

40. Pennington DL, Abé C, Batki SL, Meyerhoff DJ. A preliminary examination of cortical neurotransmitter levels associated with heavy drinking in posttraumatic stress disorder. Psychiatry Res, 2014, 224(3):281-7.

41. Hill MN, Campolongo P, Yehuda R, Patel S. Integrating Endocannabinoid Signaling and Cannabinoids into the Biology and Treatment of Posttraumatic Stress Disorder. Neuropsychopharmacology, 2018, 43(1):80-102

42. Bitencourt RM, Pamplona FA, Takahashi RN. Facilitation of contextual fear memory extinction and anti-anxiogenic effects of AM404 and cannabidiol in conditioned rats. Eur Neuropsychopharmacol, 2008, 18(12):849-59.

43. Assareh N, Gururajan A, Zhou C, Luo JL, Kevin RC, Arnold JC.
Cannabidiol disrupts conditioned fear expression and cannabidiolic acid reduces trauma-induced anxiety-related behaviour in mice. Behav Pharmacol, 2020, 31(6):591-596.

44. García-Gutiérrez MS, Navarrete F, Gasparyan A, Austrich-Olivares A, Sala F, Manzanares J. Cannabidiol: A Potential New Alternative for the Treatment of Anxiety, Depression, and Psychotic Disorders. Biomolecules, 2020, 10(11):1575.

45. Elms L, Shannon S, Hughes S, Lewis N. Cannabidiol in the Treatment of Post-Traumatic Stress Disorder: A Case Series. J Altern Complement Med. 2019, 25(4):392-397.

46. Berardi A, Schelling G, Campolongo P. The endocannabinoid system and Post Traumatic Stress Disorder (PTSD): From preclinical findings to innovative therapeutic approaches in clinical settings, Pharmacol Res. 2016, 111:668-678.

47. Bitencourt RM, Takahashi RN. Cannabidiol as a Therapeutic Alternative for Post-traumatic Stress Disorder: From Bench Research to Confirmation in Human Trials. Front Neurosci, 2018, 12:502.

48. Cannabidiol (CBD) Critical Review Report, World Health Organization, Expert Committee on Drug Dependence, Geneva, available at CANNABIDIOL (CBD) (who.int), accessed on 9th of June, 2021.

49. Britch SC, Babalonis S, Walsh SL. Cannabidiol: pharmacology and therapeutic targets. Psychopharmacology (Berl), 2021, 238(1):9-28.

50. Sartori SB, Singewald N. Novel pharmacological targets in drug development for the treatment of anxiety and anxiety-related disorders. Pharmacol Ther, 2019, 204:107402.

51. Cameron C, Watson D, Robinson J. Use of a synthetic cannabinoid in a correctional population for posttraumatic stress disorder-related insomnia and nightmares, chronic pain, harm reduction, and other indications: a retrospective evaluation. J Clin Psychopharmacol, 2014, 34(5):559-64.

52. Spindle TR, Cone EJ, Goffi E et al. Pharmacodynamic effects of vaporized and oral cannabidiol (CBD) and vaporized CBD-dominant cannabis in infrequent cannabis users. Drug Alcohol Depend, 2020, 211:107937.

53. Young MB, Norrholm SD, Khoury LM et al. Inhibition of serotonin transporters disrupts the enhancement of fear memory extinction by 3,4-methylenedioxymethamphetamine (MDMA). Psychopharmacology (Berl), 2017, 234(19):2883-2895.

54. Feduccia AA, Jerome L, Yazar-Klosinski B, Emerson A, Mithoefer MC, Doblin R. Breakthrough for Trauma Treatment: Safety and Efficacy of MDMA-Assisted Psychotherapy Compared to Paroxetine and Sertraline. Front Psychiatry. 2019 Sep 12; 10:650.

55. Mithoefer MC, Wagner MT, Mithoefer AT, Jerome L, Doblin R. The safety and efficacy of $\{+/-\} 3,4-$ methylenedioxymethamphetamineassisted psychotherapy in subjects with chronic, treatment-resistant posttraumatic stress disorder: the first randomized controlled pilot study. J Psychopharmacol, 2011, 25(4):439-52.

56. Mithoefer MC, Wagner MT, Mithoefer AT et al. Durability of improvement in post-traumatic stress disorder symptoms and absence of harmful effects or drug dependency after 3,4-methylenedioxymethamphetamineassisted psychotherapy: a prospective long-term follow-up study. J Psychopharmacol, 2013, 27(1):28-39.

57. Jerome L, Feduccia AA, Wang JB et al. Long-term follow-up outcomes of MDMA-assisted psychotherapy for treatment of PTSD: a longitudinal pooled analysis of six phase 2 trials. Psychopharmacology (Berl), 2020 237(8):2485-2497.

58. Illingworth BJ, Lewis DJ, Lambarth AT et al. A comparison of MDMA-assisted psychotherapy to non-assisted psychotherapy in treatment-resistant PTSD: A systematic review and meta-analysis. J Psychopharmacol, 2021, 35(5):501-511.

59. Schenk S, Newcombe D. Methylenedioxymethamphetamine (MDMA) in Psychiatry: Pros, Cons, and Suggestions. J Clin Psychopharmacol, 2018, 38(6):632-638.

60. Morgan L. MDMA-assisted psychotherapy for people diagnosed with treatment-resistant PTSD: what it is and what it isn't. Ann Gen Psychiatry, 2020, 19:33.

61. Jîtcă G, Ősz BE, Tero-Vescan A, Vari CE. Psychoactive Drugs-From Chemical Structure to Oxidative Stress Related to Dopaminergic Neurotransmission. A Review, Antioxidants (Basel), 2021,10(3):381.

62. de la Torre R, Farré M. Neurotoxicity of MDMA (ecstasy): the limitations of scaling from animals to humans. Trends Pharmacol Sci, 2004, 25(10):505-8. 